\title{
Phylogenetic Analyses of Four Chinese Endemic Wheat Landraces Based on Two Single Copy Genes
}

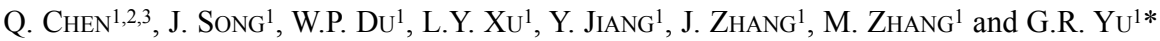 \\ ${ }^{1}$ Institute of Biotechnology and Nuclear Technology, Sichuan Academy of Agricultural Sciences, \\ Chengdu 610061, Sichuan, China \\ ${ }^{2}$ Triticeae Research Institute, Sichuan Agricultural University, Wenjiang 611130, Chengdu, Sichuan, China \\ ${ }^{3}$ Key Laboratory of Crop Genetic Resources and Improvement, Ministry of Education, \\ Sichuan Agricultural University, Wenjiang 611130, Chengdu, Sichuan, China
}

(Received 8 March 2017; Accepted 11 October 2017;

Communicated by T. Harangozó and R.A. McIntosh)

\begin{abstract}
Chinese endemic wheat landraces possess unique morphological features and desirable traits, useful for wheat breeding. It is important to clarify the relationship among these landraces. In this study, 21 accessions of the four Chinese endemic wheat landrace species were investigated using single-copy genes encoding plastid Acetyl-CoA carboxylase (Acc-1) and 3-phosphoglycerate kinase $(P g k-1)$ in order to estimate their phylogenetic relationship. Phylogenetic trees were constructed using maximum parsimony (MP), maximum likelihood (ML) and Bayesian, and TCS network and gene flow values. The A and B genome sequences from the Pgk-1 loci indicated that three accessions of Triticum petropavlovskyi were clustered into the same subclade, and the T. aestivum ssp. tibetanum and the Sichuan white wheat accessions were grouped into a separate subclade. Based on the Acc-1 gene, T. aestivum ssp. tibetanum and T. aestivum ssp. yunnanense were grouped into one subclade in the A genome; the B genome from T. petropavlovskyi and T. aestivum ssp. tibetanum, and the Sichuan white wheat complex and T. aestivum ssp. tibetanum were grouped in the same clades. The $\mathrm{D}$ genome of T. aestivum ssp. yunnanense clustered with T. petropavlovskyi. Our findings suggested that (1) T. petropavlovskyi is distantly related to the Sichuan white wheat complex; (2) T. petropavlovskyi, T. aestivum ssp. tibetanum and T. aestivum ssp. yunnanense are closely related; (3) T. aestivum ssp. tibetanum is closely related to T. aestivum ssp. yunnanense and the Sichuan white wheat complex; and (4) T. aestivum ssp. tibetanum may be an ancestor of Chinese endemic wheat landraces.
\end{abstract}

Keywords: $A c c-1, P g k-1$, Chinese endemic wheat landraces, phylogenetic relationships

\section{Introduction}

Landraces cultivated around the world in the past are important genetic resources (Harlan 1975). China was proposed as a center of diversity for wheat (Yen et al. 1988). Four unique Chinese wheat landrace species described as Xinjiang rice wheat (Triticum petropavlovskyi Udacz. et Migusch.), Tibetan weedrace (T. aestivum ssp. tibetanum Shao), Yunnan hulled wheat (T. aestivum ssp. yunnanense King) and Sichuan white wheat com-

\footnotetext{
*Corresponding author; E-mail: guirongyu@yeah.net
} 
plex (T. aestivum L.) were discovered in Yunnan, Tibet, the Xinjiang Autonomous Region, and Sichuan, respectively (Dong and Zheng 2000). Cytological studies suggested that the Chinese endemic wheat landraces harbor primitive and stable AABBDD chromosomal constitutions (Shao et al. 1980; Dong et al. 1981; Chen et al. 1988; Huang et al. 1989; Chen et al. 1991), and possess unique morphological features, such as spike fragility and glume tenacity. These four unique wheat landrace species also possess desirable traits for wheat breeding, such as resistance to preharvest sprouting and tolerance to cold or heat (Chen et al. 1988; Dong et al. 1981; Shao et al. 1980). Thus, it is important to investigate the phylogenetic relationships of the four unique taxa as primary gene pools and their potentially useful traits for common wheat improvement.

Numerous studies have been conducted to elucidate the phylogenetic relationships among the four groups. Based on morphology and geographical distribution, T. petropavlovskyi was considered to be different from the other three landrace groups (Shao et al. 1980; Yen et al. 1988). In RFLP-based genetic analysis, T. petropavlovskyi was distinct from the other three Chinese landrace groups (Ward et al. 1998). T. aestivum ssp. tibetanum has a naturally broken rachis with wedge type disarticulation (Shao et al. 1980; Tsunewaki et al. 1990), the same as T. aestivu ssp. yunnanense (Shao et al. 1980; Tsunewaki et al. 1990). The Sichuan white wheat complex is composed of cultivated common wheats characterized by multifloret spikelets and rounded glumes (Yen et al. 1988). Based on distribution, morphological traits, and RFLP clustering, T. aestivum ssp. tibetanum was considered the ancestor of T. aestivum ssp. yunnanense and the Sichuan white wheat complex (Ward et al. 1998).

Despite intensive research the relationships of the four Chinese endemic landrace species remain uncertain, and few attempts have been made to study the phylogenetic relationships based on DNA sequences. The plastid acetyl-CoA carboxylase (ACCase) $($ Acc-1) and the plastid 3-phosphoglycerate kinase (PGK) (Pgk-1) genes are single-copy nuclear genes that are less susceptible to coevolution (Sang 2002; Smith et al. 2006; Chalupska et al. 2008), and therefore, useful in phylogenetic studies (Huang et al. 2002a,b; Fan et al. 2007; Golovnina et al. 2007; Yan and Sun 2011; Fan et al. 2012).

In the present research, we sequenced and analyzed the single copy Pgk-1 and Acc-1 genes, in 21 accessions representing the four endemic wheat landraces, to elucidate their phylogenetic relationships.

\section{Materials and methods}

\section{Plant materials}

The materials used in this study included 21 accessions of Chinese endemic wheat landrace species (Table S1*). The Acc-1 sequences of accessions with EU numbers were obtained from previously reported data (Kang et al. 2010). The Pgk-1 sequences of accessions with JQ numbers were obtained from our published data (Chen et al. 2013). The rest of the sequences were initially obtained in this study.

\footnotetext{
*Further details about the Electronic Supplementary Material (ESM) can be found at the end of the article.
} 


\section{DNA amplification, cloning, and sequencing}

DNA extraction followed a standard CTAB protocol (Doyle and Doyle 1987). The singlecopy gene $P g k-1$ was amplified by polymerase chain reaction (PCR) using the primer pair PgkF1 and PgkF1 (Huang et al. 2002ab). Primers AccF1 and AccF2 (Fan et al. 2009) were used to amplify Acc-1.

PCR products were cloned into the pMD19-T vector (Takara, Dalian) following the manufacturer's instructions. Cloning of PCR amplifications from a single copy gene in an allopolyploid species should enable isolation of homoeologous sequences from each genome (Doyle and Doyle 1999; Fan et al. 2009). The cloned PCR products were sequenced by the Beijing Genomics Institute.

\section{Phylogenetic reconstruction}

Multiple sequence alignments of $P g k-1$ and $A c c-1$ were conducted using Clustal X (Thompson et al. 1999), with manual adjustment. To reduce the size of the matrices and possible effects of PCR artifacts (Cronn et al. 2002), unique substitutions in single clones were ignored. Sequences generated in this study were submitted to GenBank and the accession numbers are provided in Table S1.

The sequence statistics, including nucleotide substitutions and transition/transversion (TS/TV) ratio were calculated using MEGA 6 (Tamura et al. 2011; Tamura et al. 2013). Nucleotide diversity, gene flow and genetic differentiation $\left(G_{S T}\right)$ in each accession were analyzed using DnaSP (Librado and Rozas 2009). Tests of neutrality were performed as described by Tajima (1989).

Phylogenetic analysis using maximum parismony (MP) was performed for Pgk-1 using the computer program PAUP* beta version 4.0b10 (Swofford D L, Sinauer Associates, http://www.sinauer.com). All characters were specified as unweighted and unordered, and gaps were excluded from the analyses. The most parsimonious trees were obtained by performing a heuristic search using the Tree Bisection-Reconnection (TBR) option with MulTrees, and 10 replications of random addition sequences with the stepwise addition option. Multiple parsimonious trees were combined to form a strict consensus tree. The overall character congruence was estimated by consistency index (CI), and retention index (RI). To infer the robustness of clades, bootstrap values with 1,000 replications (Felsenstein 1985) were calculated by performing a heuristic search using the TBR option with Multrees.

Maximum likelihood (ML) and Bayesian analyses were performed for Pgk-1 and Acc-1 using PhyML v3.0 for ML (Guindon et al. 2010; Guindon and Gascuel 2003) and MrBayes v3.2.2 for Bayesian (Huelsenbeck and Ronquist 2001; Ronquist and Huelsenbeck 2003), respectively. The nucleotide substitution models used in the ML method were estimated by Modeltest v3.7 using the Akaike Information Criterion (AIC) (Posada and Crandall 1998). The HKY $+\mathrm{I}+\mathrm{G}$ model produced the highest log-likelihood value for Acc- 1 data, and the best-fit model was identified as GTR + G for Pgk-1 data. ML heuristic searches were performed with 100 random addition sequence replications and TBR 
branch swapping algorithm. The robustness of the trees was estimated by bootstrap supports (BS) (Felsenstein 1985).

For Bayesian analysis, MrModeltest v2.3 was used to test different models of sequence evolution, and the HKY $+\mathrm{I}+\mathrm{G}$ model was chosen by AIC for Acc-1 and GTR $+\mathrm{G}$ for $P g k-1$. Analyses were performed with 2,000,000 generations of one cold and three heated Markov Chain Monte Carlo chains, sampling trees every 100 generations. The first 500 trees were discarded as "burn-in" and the remaining trees were used to construct the 50\% majority rule consensus tree. Statistical confidence in nodes was evaluated by posterior probabilities (PP).

\section{TCS analyses}

The Templeton, Crandall, Sing (TCS) reconstruction method is an effective method to reveal specific progenitor descendant relationships in perennial Triticeae (Chen et al. 2013; Luo et al. 2012; Yan and Sun 2011), and thus it was employed to elucidate the number of halotypes of the Acc-1 and Pgk-1 sequences, and their relatedness. Before reconstructing the TCS networks, a test of recombination was performed using the Phi (pairwise homoplasy index) method with Splits Tree (Huson and Bryant 2006). Building upon this test, the $A c c-1(\mathrm{P}=0.9019)$ and Pgk-1 ( $\mathrm{P}=0.9838)$ sequences were used to generate the TCS networks. The TCS haplotype network was tested to evaluate possible genetic relationships between haplotypes with the computer program TCS 1.2.1 (Clement et al. 2000).

\section{Results}

\section{Sequence characteristics}

The characteristics of the Acc-1 gene are shown in Table S2. Complete alignment of Acc-1 sequences confirmed a $46 \mathrm{bp}$ insertion/deletion (indel) at position 1160-1207. This 46 bp deletion occurred in $A c c-1$ alleles in A and D genomes in all accessions.

The characteristics of the $P g k-1$ gene are shown in Table S2. Many gaps resulting from indels were found in alignment of the $P g k-1$ sequence data. In particular, apart from single nucleotide substitutions and deletions, two insertions and one deletion occurred in the A genome. Firstly, a 6 bp insertion (TCCACT) was found at position 63-70, and a 2 bp TA insertion was present at position 487-490. In addition, a 4 bp deletion (AACC) occurred at the position 569-574 in the A genome.

\section{Gene flow and genetic differentiation $\left(G_{S T}\right)$ of the four endemic wheat landrace species}

Based on the Acc-1 gene, the $G_{S T}$ values among T. aestivum ssp. tibetanum, T. aestivum ssp. yunnanense and T. petropavlovskyi were less than zero. The $G_{S T}$ values between the Sichuan white wheat complex and each of the other three landrace groups were greater than zero (Table S3). Moreover, the $G_{S T}$ values for Pgk-1 sequences among the four groups were greater than zero (Table S3). 


\section{Phylogenetic analyses of Acc-1 sequences}

The aligned Acc- 1 sequences contained 143 variable sites, of which 23 were parsimonyinformative. ML analysis resulted in a single phylogenetic tree $(-\operatorname{Lnlikelihood}=3039.30)$ with assumed nucleotide frequencies A: 0.2438, C: 0.1936, G: 0.2200, and T: 0.3427, and gamma shape parameter $=0.8410$. Bayesian analysis inferred the same topology as the ML analysis. The tree illustrated in Fig. S1 is the ML tree with BS above and PP below branches.

Phylogenetic analyses clearly separated the sequences of the Acc-1 gene into two major clades, i.e., Clades I and II. Clade I included sequences of the A and B genomes, and Clade II included genome D. In Clade I, A genome-specific sequences from T. petropavlovskyi accessions (except AS362), T. aestivum ssp. tibetanum, T. aestivum ssp. yunnanense (except AS311), and the Sichuan white wheat complex (T. aestivum cv. Chinese Spring and T. aestivum cv. J-11) formed a group with 99\% PP support, and one T. aestivum ssp. tibetanum accession (AS1026) together with one accession of T. aestivum ssp. yunnanense (AS343) formed a subclade. The sequences from the B genome mapped one major subclade, with 54\% BS and 100\% PP. In this subclade, one accession of T. aestivum ssp. tibetanum (AS330) and T. aestivum cv. J-11 were grouped into the same subclade (97\% PP). In Clade II, two T. aestivum ssp. yunnanense accessions (AS331 and AS332) together with three accessions of T. petropavlovskyi (AS356, AS358, and AS360) were placed in the same group.

\section{Phylogenetic analyses of Pgk-1 sequences}

Maximum parismony analysis using Hordeum vulgare as the outgroup was conducted (CI $=0.6935 ; \mathrm{RI}=0.9718)$. A separate $\mathrm{ML}$ analysis using the GTR $+\mathrm{G}$ model resulted in a single tree with a mean log-likelihood value of -3159.59 , and assumed nucleotide frequencies A: $0.2592, \mathrm{C}: 0.1967, \mathrm{G}: 0.2375$, and $\mathrm{T}: 0.3067$, and gamma shape parameter $=$ 0.4490. The tree topologies were almost identical in both ML and Bayesian trees, and were similar to those generated by MP with minor differences. One of the most parsimonious trees with BS (above) and Bayesian PP (below) is shown in Fig. S2.

Phylogenetic analyses clearly separated sequences (A, B, and D genomes) into Clades I and II. Clade I contained all of the A genome sequences, except for T. aestivum cv. Chinese Spring, which clustered into one subclade with high statistical support $(98 \% \mathrm{BS}$ and $100 \%$ PP) (subclade A). Within Subclade A, three accessions of T. petropavlovskyi (AS356, AS358, and AS363) formed a group with 92\% BS and 96\% PP, and T. aestivum cv. J-11 and T. aestivum ssp. tibetanum (AS330) grouped into the same group with $80 \%$ BS. Clade II included the B and D genome sequences. Two subclades (subclades B and D) with high statistical support were included in this clade. Subclade B included all the B genome sequences, among which T. aestivum cv. Chinese Spring, T. aestivum cv. J-11 and T. aestivum ssp. tibetanum (AS1027) clustered into the same clade with $79 \% \mathrm{BS}$; and three T. petropavlovskyi (AS358, AS359 and AS360) formed a group with 95\% BS and $100 \%$ PP. Subclade D consisted of sequences from the D genome with no clustering. 


\section{TCS analyses}

The TCS procedure used to analyze haplotype relationships among the Triticum accessions in this study (Sun et al. 2009) defined a 95\% parismony connection limit of 13 steps for exon alignment of the Acc-1 (Fig. S3) and Pgk-1 genes (Fig. S4). The TCS network consisted of three major haplotype groups corresponding to the $\mathrm{A}, \mathrm{B}$, and $\mathrm{D}$ genomes (Fig. S3 and S4). The length of the branches between two nodes was meaningless here and each one implied one nucleotide difference. There were several differences among the TCS haplotype networks, the ML tree for Acc-1, and the Bayesian tree for Pgk-1. Figure S3 shows that the difference in the A genome haplotype network was that T. petropavlovskyi (AS362) exhibited a similar haplotype to T. aestivum ssp. yunnanense (AS331). Two differences between the TCS and ML tree of the B genome were observed. Firstly, T. petropavlovskyi (AS362) connected with T. aestivum ssp. yunnanense (AS343); secondly, the T. aestivum cv. J-11 and T. aestivum ssp. tibetanum (AS330) group appeared to be related to the group including T. petropavlovskyi (AS359) and T. aestivum ssp. tibetanum (AS1026). The network of the D genome was similar to the ML tree that included two T. petropavlovskyi accessions (AS356 and AS360) and had a similar haplotype to T. aestivum ssp. yunnanense (AS332).

In Figure S4, haplotype groups (A, B, and D) were similar in the Bayesian tree from the Pgk-1 sequences. The network of the A genome indicated a much closer relationship between T. aestivum cv. J-11 and T. aestivum ssp. tibetanum, similar to the Bayesian analysis. However, a group including three accessions of T. petropavlovskyi (AS356, AS358, and AS363) was unavailable in the haplotype network of the A genome. For the B genome, the haplotype of T. aestivum cv. Chinese Spring, T. aestivum cv. J-11, and T. aestivum ssp. tibetanum (AS1027) appeared to be related in the phylogenetic tree. The difference between the Bayesian analysis and TCS was a cluster with three T. petropavlovskyi (AS358, AS359, and AS360), which was not detected in the TCS network.

\section{Discussion}

\section{Genetic diversity of four Chinese endemic wheat landrace species}

Several studies of genetic diversity among Chinese endemic wheat landrace sepcies have been reported. Wei et al. $(2001,2002)$ analyzed genetic diversity using A-PAGE, SDSPAGE, STS-PCR, and SSR markers in 32 accessions of unique Chinese endemic wheat landrace species. The results indicated higher genetic diversity for T. petropavlovskyi and T. aestivum ssp. tibetanum than for T. aestivum ssp. yunnanense. Based on RFLP analysis, Ward et al. (1998) found higher genetic diversities for T. petropavlovskyi and T. aestivum ssp. tibetanum than for T. aestivum ssp. yunnanense. However, analysis of an esterase isozyme revealed that $T$. aestivum ssp. yunnanense had high genetic diversity (Cui and Ma 1991). Akond et al. (2005) indicated that genetic polymorphism of T. petropavlovskyi was low. Furthermore, Wang et al. (2007) considered that T. petropavlovskyi had relatively low SSR variation. In the present study, many indels (nucleotide substitutions, insertions and deletions) were detected in the Acc-1 and Pgk-1 sequences among the four 
Chinese endemic wheat landraces, confirming high nucleotide diversity. The different results concerning genetic diversity of Chinese endemic wheat landrace species might be influenced by sample size, different accessions, and different types of molecular markers used in each study.

Relationships between T. petropavlovskyi and the other three Chinese endemic wheat landrace species

Based on morphological traits, chromosome pairing behavior, and geographical trait, T. petropavlovskyi was considered to be distinct from the other taxa (Shao et al. 1980; Chen et al. 1988; Yen et al. 1988). Previous studies indicated that differences between T. petropavlovskyi and the Sichuan white wheat complex were related to the B genome (Yao et al. 1983; Chen et al. 1985; Chen et al. 1988). Based on HMW-glutenin and gliadin variations, Wei et al. (2002) suggested that Glu-B1 differed between T. petropavlovskyi and the Sichuan white wheat. Furthermore, using RFLP, Ward et al. (1998) inferred that T. petropavlovskyi differed from the other Chinese wheat landraces. The results of SSR and EST-SSR markers indicated that T. petropavlovskyi was significantly different from the other three landrace groups in the A, B, and D genomes (Yang et al. 2005).

In the present study, Pgk-1 sequences of the A and B genomes of T. petropavlovskyi clustered into the same subclade, indicating that $T$. petropavlovskyi is distantly related to the other three groups. However, the phylogenetic tree of Acc-1 showed that T. petropavlovskyi was clustered with T. aestivum ssp. tibetanum and T. aestivum ssp. yunnanense in the B and D genomes, respectively. The results of $G_{\mathrm{ST}}$ among T. petropavlovskyi, T. aestivum ssp. tibetanum and T. aestivum ssp. yunnanense were less than zero indicating that these three landrace groups had a close genetic relationship. Overall, our results revealed a closer relationship among T. petropavlovskyi, T. aestivum ssp. tibetanum and T. aestivum ssp. yunnanense than between T. petropavlovskyi and the Sichuan white wheat complex.

\section{Relationship among T. aestivum ssp. tibetanum, T. aestivum ssp. yunnanense,} and the Sichuan white wheat complex

Because of semi-wild traits, geographical distribution, and morphological similarities, T. aestivum ssp. tibetanum was presumed to be the ancestor of T. aestivum ssp. yunnanense and the Sichuan white wheat complex (Chen et al. 1988; Yen et al. 1988; Yang et al. 1992). RFLP analysis revealed that $T$. aestivum ssp. tibetanum was genetically close to T. aestivum ssp. yunnanense and the Sichuan white wheat complex (Ward et al. 1998). Cluster analysis based on genetic distance $(G D)$ indicated a closer relationship between T. aestivum ssp. tibetanum and T. aestivum ssp. yunnanense (Wang et al. 2007).

In the present research, the $G_{S T}$ values of the $A c c-1$ and $P g k-1$ sequences between T. aestivum ssp. yunnanense and T. aestivum ssp. tibetanum were low, indicating a close genetic relationship. Phylogenetic analyses of the Pgk-1 gene showed that T. aestivum ssp. tibetanum and the Sichuan white wheat complex were clustered in one group based on the A and B genomes. The phylogenetic tree of Acc-1 showed that T. aestivum ssp. 
yunnanense and T. aestivum ssp. tibetanum were grouped together in the A genome, and T. aestivum ssp. tibetanum and the Sichuan white wheat complex were grouped in the B genome, a result that was congruent with the TCS analysis. These results suggested that the relationships among $T$. aestivum ssp. tibetanum, $T$. aestivum ssp. yunnanense and the Sichuan white wheat complex are close.

\section{Acknowledgments}

This work was financially supported by the Applied and Foundational Program of Science and Technology Bureau of Sichuan province (2015JY0019).

\section{References}

Akond, A.S.M.G.M., Watanabe, N., Furuta, Y. 2005. Genetic variation among Portuguese landraces of 'Arrancada' wheat and Triticum petropavlovskyi by AFLP-based assessment. Genet. Resour. Crop Evol. 52:619-628.

Chalupska, D., Lee, H.Y., Faris, J.D., Evrard, A., Chalhoub, B., Haselkorn, R., Gornicki, P. 2008. Acc homoeoloci the evolution of wheat genomes. Proc. Natl. Acad. Sci. 105:9691-9696.

Chen, P.D., Huang, L., Liu, D. J. 1991. Analysis of the genomic constitution of Xizang wheat (Triticum aestivum ssp. tibetanum Shao) using double ditelosomics of T. aestivum cv. Chinese Spring. Acta. Genet. Sin. 18:39-43.

Chen, P.D., Liu, D.J., Pei, G.Z., Qi, L.L., Huang, L. 1988. The chromosome constitution of three endemic hexaploid wheats in western China. In: Miller, T.E., Koebner R.M.D. (eds), Proc. 7th Int. Wheat Genet. Symp. Cambridge, U.K. pp. 75-80.

Chen, Q., Sun, Y.Z., Dong, Y.S. 1985. Cytogenetical Studies on Interspecific Hybrids of Xinjiang wheat. Acta. Agron. Sin. 11:23-28.

Chen, Q., Kang, H.Y., Fan, X., Wang, Y., Sha, L.N., Zhang, H.Q., Zhong, M.Y., Xu, L.L., Zeng, J., Yang, R.W., Zhang, L., Ding, C.B., Zhou, Y.H. 2013. Evolutionary history of Triticum petropavlovskyi Udacz. et Migusch. inferred from the sequences of the 3-phosphoglycerate kinase gene. PloS One 8:e71139.

Clement, M., Posada, D., Crandall, K.A. 2000. TCS: a computer program to estimate gene genealogies. Mol. Ecol. 9:1657-1659.

Cronn, R., Cedroni, M., Haselkorn, T., Grover, C., Wendel, J.F. 2002. PCR-mediated recombination in amplification products derived from polyploid cotton. Theor. Appl. Genet.104: 482-489.

Cui, Y.X., Ma, Y. 1991. Esterase isozyme of Chinese endemic wheat. Acta. Bot. Sin. 32:39-44.

Dong, Y.S. 2000. Wheat genetic resources in China. In: Dong, Y.S., Zheng, D.S. (eds), Wheat Genetic Resources in China. China Agriculture Press. Beijing, China. pp. 16-30.

Dong, Y.S., Zheng, D.S., Qiao, D.Y., Zeng, X.Q., En, Z.C., Chen, X.R. 1981. Investigation and study on Yunnan wheat (Triticum aestivum ssp. yunanense King). Acta. Agron. Sin. 7:145-151.

Doyle, J.J., Doyle, J.L. 1987. A rapid DNA isolation procedure for small quantities of fresh leaf tissue. Phytochem. Bull. 19:11-15.

Doyle, J.J., Doyle, J.L. 1999. Nuclear Protein-coding Genes in Phylogeny Reconstruction and Homology Assessment. In: Hollingsworth, P.M., Bateman, R.M., Gornall, R.J. (eds), Molecular Systematics and Plant Evolution. Taylor \& Francis Press, pp. 229-254.

Fan, X., Sha, L.N., Yang, R.W., Zhang, H.Q., Kang, H.Y., Ding C.B., Zhang, L., Zheng, Y.L., Zhou, Y.H. 2009. Phylogeny and evolutionary history of Leymus (Triticeae; Poaceae) based on a single-copy nuclear gene encoding plastid acetyl-CoA carboxylase. BMC Evol. Biol. 9:247.

Fan, X., Sha, L.N., Zeng, J., Kang, H.Y., Zhang, H.Q., Wang, X.L., Zhang, L., Yang, R.W., Ding, C.B., Zheng, Y.L., Zhou, Y.H. 2012. Evolutionary dynamics of the Pgk1 gene in the polyploid genus Kengyilia (Triticeae: Poaceae) and its diploid relatives. PloS One 7:e31122. 
Fan, X., Zhang, H.Q., Sha, L.N., Zhang, L., Yang, R.W., Ding, C.B., Zhou, Y.H. 2007. Phylogenetic analysis among Hystrix, Leymus and its affinitive genera (Poaceae: Triticeae) based on the sequences of a gene encoding plastid acetyl-CoA carboxylase. Plant Sci. 172:701-707.

Felsenstein, J. 1985. Confidence limits on phylogenies: an approach using the bootstrap. Evolution 39:783-791.

Golovnina, K.A., Glushkov, S.A., Blinov, A.G., Mayorov, V.I., Adkison, L.R., Goncharov, N.P. 2007. Molecular phylogeny of the genus Triticum L.. Plant Syst. Evol. 264:195-216.

Guindon, S., Dufayard, J.F., Lefort, V., Anisimova, M., Hordijk, W., Gascuel, O. 2010. New algorithms and methods to estimate maximum-likelihood phylogenies: assessing the performance of PhyML 3.0. Syst. Biol. 59:307-321.

Guindon, S., Gascuel, O. 2003. A simple, fast, and accurate algorithm to estimate large phylogenies by maximum likelihood. Syst. Biol. 52:696-704.

Harlan, J.R. 1975. Our vanishing genetic resources. Science 188:617-621.

Huang, L., Chen, P.D., Liu, D.J. 1989. Analysis of the genomic constitution of Yunnan wheat (Triticum aestivum ssp. yunnanese King) using double ditelosomics of T. aestivum cv. Chinese Spring. Sci. Agri. Sin. 22:13-16.

Huang, S., Sirikhachornkit, A., Faris, J.D., Su, X., Gill, B.S., Haselkorn, R., Gornicki, P. 2002a. Phylogenetic analysis of the acetyl-CoA carboxylase and 3-phosphoglycerate kinase loci in wheat and other grasses. Plant Mol. Biol. 48:805-820.

Huang, S., Sirikhachornkit, A., Su, X., Fairs, J., Gill, B., Haselkorn, R., Gornicki, P. 2002b. Genes encoding plastid acetyl-CoA carboxylase and 3-phosphoglycerate kinase of the Triticum/Aegilops complex and the evolutionary history of polyploid wheat. Proc. Natl. Acad. Sci. USA. 99:8133-8138.

Huelsenbeck, J.P., Ronquist, F. 2001. MRBAYES: Bayesian inference of phylogenetic trees. Bioinformatics 17:754-755.

Huson, D.H., Bryant, D. 2006. Application of phylogenetic networks in evolutionary studies. Mol. Biol. Evol. 23:254-267.

Kang, H.Y., Fan, X., Zhang, H.Q., Sha, L.N., Sun, G., Zhou, Y.H. 2010. The origin of Triticum petropavlovskyi Udacz. et Migusch.: demonstration of the utility of the genes encoding plastid acetyl-CoA carboxylase sequence. Mol. Breeding 25:381-395.

Librado, P., Rozas, J. 2009. DnaSP v5: a software for comprehensive analysis of DNA polymorphism data. Bioinformatics 25:1451-1452.

Luo, X., Tinker, N.A., Fan, X., Zhang, H.Q., Sha, L.N., Kang, H.Y., Ding, C.B., Liu, J., Zhang, L., Yang, R.W., Zhou, Y.H. 2012. Phylogeny and maternal donor of Kengyilia species (Poaceae: Triticeae) based on three cpDNA (matK, rbcL and trnH-psbA) sequences. Biochem. Syst. Ecol. 44:61-69.

Posada, D., Crandall, K.A. 1998. Modeltest: testing the model of DNA substitution. Bioinformatics 14:817818.

Ronquist, F., Huelsenbeck, J.P. 2003. MrBayes 3: Bayesian phylogenetic inference under mixed models. Bioinformatics 19:1572-1574.

Sang, T. 2002. Utility of low-copy nuclear gene sequences in plant phylogenetic. Crit. Rev. Biochem. Mol. Bio.1 37:121-147.

Shao, Q.Q., Li, C.S., Basang, C.R. 1980. Semi-wild wheat from Xizang (Tibet). Acta. Genet. Sin. 7:150-156.

Smith, J., Funke, M., Woo, V. 2006. A duplication of $g c y c$ predates divergence within tribe Coronanthereae (Gesneriaceae): phylogenetic analysis and evolution. Plant syst. Evol. 261:245-256.

Sun, G.L., Salomon B. 2009. Molecular evolution and origin of tetraploid Elymus species. Breeding Sci. 59:487-491.

Tajima, F. 1989. Statistical method for testing the neutral mutation hypothesis by DNA polymorphism. Genetics 123:585-595.

Tamura, K., Peterson, D., Peterson, N., Stecher, G., Nei, M., Kumar, S. 2011. MEGA5: molecular evolutionary genetics analysis using maximum likelihood, evolutionary distance, and maximum parsimony methods. Mol. Biol. Evol. 28:2731-2739.

Tamura, K., Stecher, G., Peterson, D., Filipski, A., Kumar, S. 2013. MEGA6: Molecular evolutionary genetics analysis version 6.0. Mol. Biol. Evol. 30:2725-2729.

Thompson, J.D., Plewniak, F., Poch, O. 1999. A comprehensive comparison of multiple sequence alignment programs. Nucl. Acids. Res. 27:2682-2690. 
Tsunewaki, K., Yamada, S., Mori, N. 1990. Genetical studies on a Tibetan semi-wild wheat, Triticum aestivum ssp. tibetanum. Jpn. J. Genet. 65:353-365.

Wang, H.Y., Wang, X.E., Chen, P.D., Liu, D.J. 2007. Assessment of Genetic Diversity of Yunnan, Tibetan, and Xinjiang Wheat Using SSR Markers. J. Genet. Genom. 34:623-633.

Ward, R.W., Yang, Z.L., Kim, H.S., Yen, C. 1998. Comparative analyses of RFLP diversity in landraces of Triticum aestivum and collections of T. tauschii from China and Southwest Asia. Theor. Appl. Genet. 96:312-318.

Wei, Y.M., Zheng, Y.L., Liu, D.C., Zhou, Y.H., Lan, X.J. 2002. HMW-glutenin and gliadin variations in Tibetan weedrace, Xinjiang rice wheat and Yunnan hulled wheat. Genet. Resour. Crop Evol. 49:327-330.

Wei, Y.M., Zheng, Y.L., Zhou, Y.H., Liu D.C., Lan, X.J., Yan, Z.H., Zhang, Z.Q. 2001. Genetic diversity of Gli-1, Gli-2 and Glu-1 alleles among Chinese endimic wheat. Acta. Bot. Sin. 43:834-839.

Yang, W.Y., Yen, C., Yang, J.L. 1992. Cytogenetic study on the origin of some special Chinese landraces of common wheat. Wheat Inform. Serv. 75:14-20.

Yang, X.Q., Peng, L., Han, Z.F., Ni, Z.F., Sun, Q.X. 2005. Genetic diversity revealed by genomic-SSR and EST-SSR markers among common wheat, spelt and compactum. Prog. Nat. Sci. 15:24-33.

Yan, C., Sun, G.L. (2011) Nucleotide divergence and genetic relationships of Pseudoroegneria species. Biochem. Syst. Ecol. 39:309-319.

Yao, J.X., Yang, F.B., Shi, S.Y., Zhao, Y.M. 1983. Research on a new species in Triticum-Xinjiang wheat with rice-like spike. Hereditas 5:17-20.

Yen, C., Yang, J.L., Luo, M.C. 1988. The origin of the Tibetan weedrace of hexaploid wheat, Chinese Spring, Chengdu guangtou and other landraces of white wheat complex from china. In: Miller, T.E., Koebner R.M.D. (eds), Proc. 7th Int. Wheat Genet. Symp. Cambridge, U.K. pp. 175-179.

\section{Electronic Supplementary Material (ESM)}

Electronic Supplementary Material (ESM) associated with this article can be found at the website of CRC at http://www.akademiai.com/content/120427/

Electronic Supplementary Table S1. Plants used in this study

Electronic Supplementary Table S2. Characteristics derived from Acc-1 and Pgk-1 sequences

Electronic Supplementary Figure S1. Maximum-likelihood tree from the Acc-1 gene of Chinese endemic wheat landraces. Numbers above nodes are bootstrap values $\geq 50 \%$; numbers below nodes are posterior probability values $\geq 90 \%$; Genome composition, species name and accession number/cultivar name are indicated for each

taxon

Electronic Supplementary Figure S2. Maximum parismony tree from the $P g k-1$ gene of Chinese endemic wheat landraces. Numbers above nodes are bootstrap values $\geq 50 \%$; numbers below nodes are posterior probability values $\geq 90 \%$; Genome composition, species name and accession number/cultivar name are indicated for each taxon

Electronic Supplementary Figure S3. TCS network inferred from the Acc-1 gene of Chinese endemic wheat landraces. Accession numbers of each species are listed in Table 1. Haplotypes in the network are represented by circles of different color corresponding to the different Chinese endemic wheat landraces; the small circle in the lineage chains represents SNP changes

Electronic Supplementary Figure S4. TCS network inferred from the Pgk-1 gene of Chinese endemic wheat landraces. Accession numbers of each species are listed in Table 1. Haplotypes in the network are represented by circles of different color corresponding to the different Chinese endemic wheat landraces; the small circle in the lineage chains represents SNP changes 